\title{
Informal carers' experience and outcomes of assistive technology use in dementia care in the community: a systematic review protocol
}

Vimal Sriram $^{*} \mathbb{B}$, Crispin Jenkinson and Michele Peters

\begin{abstract}
Background: Dementia is one of the greatest health and care priorities globally. Caring for persons with dementia is a challenge and often leads to negative psychological, physiological and financial consequences for informal carers (family members or friends). Many informal carers experience moderate to severe levels of burden. Advances in technology have the potential to assist persons with dementia and their carers, through assistive technology (AT) devices such as electronic medication dispensers, robotic devices and motion detectors. However, little is known about informal carers' experience and the impact of these technologies on them. This review aims to investigate the outcomes and experience of carers of persons with dementia, who live at home and use AT.

Method: MEDLINE, Embase, CINAHL, AMED, ALOIS, PsycINFO, Trial registries and OpenGrey databases will be searched for studies of any design that have investigated carer experience and/or outcomes of AT use for persons with dementia living at home. Manual searches from reference lists of relevant papers will also be undertaken. Outcomes of interest are carers' self-reported outcomes (which include perceived burden, quality of life and wellbeing) and carer experiences (such as usefulness, benefits and disadvantages of AT and impact on caregiver/care receiver relationship). Two independent reviewers will screen identified papers with pre-defined eligibility criteria and extract data using a bespoke extraction form. Discrepancies will be resolved in discussion with a third reviewer. A synthesis of eligible studies and summary will be provided.

Discussion: A systematic review of quantitative, qualitative and mixed methods evidence of informal carers' experience of AT use in dementia in the community will be carried out. It is anticipated that this will highlight (1) investigations on impact of AT use on carers, (2) outcome measures and experience questionnaires that have been used and (3) the types of studies carried out so far on this topic. The results from the review will be presented in a summary matrix of common types (e.g. mobile phones, alarms) and uses (e.g. communication, safety, personal care) of AT in dementia care and also identify AT that is not usually available through government or health system funding.
\end{abstract}

Systematic review registration: PROSPERO CRD42017082268.

Keywords: Dementia, Informal carer, Assistive technology, Quality of life, Stress, Burden

\footnotetext{
* Correspondence: vimal.sriram@dph.ox.ac.uk

Health Services Research Unit, Nuffield Department of Population Health,

University of Oxford, Richard Doll Building, Old Road Campus, Oxford OX3

7LF, UK
}

(c) The Author(s). 2019 Open Access This article is distributed under the terms of the Creative Commons Attribution 4.0 International License (http://creativecommons.org/licenses/by/4.0/), which permits unrestricted use, distribution, and reproduction in any medium, provided you give appropriate credit to the original author(s) and the source, provide a link to the Creative Commons license, and indicate if changes were made. The Creative Commons Public Domain Dedication waiver (http://creativecommons.org/publicdomain/zero/1.0/) applies to the data made available in this article, unless otherwise stated. 


\section{Background}

Dementia is becoming increasingly prevalent as the population ages and has been declared a public health priority [1]. There are an estimated 46.8 million people living with dementia globally, and the number is likely to double by 2035 [2]. Most of the care for persons with dementia living at home is provided by informal cares [3]. In the UK, there are over 700,000 informal carers for persons living with dementia [4]. Reports estimate the value of time given by informal carers to persons with dementia at approximately $£ 12.4$ billion a year for the UK $[4,5]$, and the global economic cost of dementia is estimated to be over US\$ 604 billion [6]. In England, about $15 \%$ of dementia carers say they are not in work because of their caring responsibilities and around 39\% of carers spend more than $100 \mathrm{~h}$ per week caring for a person with dementia [7].

Caring for an adult with dementia impacts on psychological, financial, economic and physical wellbeing of the carer [8-12]. Without support from informal carers, any formal support system for a person with dementia is likely to collapse. Caring for a person with dementia is not a static process [13], there is currently no cure for dementia and it is progressive; the trajectory of progress is uncertain, and carers are faced with the complexity of not knowing how quickly the dementia will progress. Therefore, it is vital to support carers in their experiences of providing care. In the UK, a national strategy for carers [14-16] is committed to supporting carers at an early stage and recognising the value of their contribution. Furthermore, the Care Act [17] sets out requirements for assessment and support centred around carers' wellbeing. These, however, are poorly adopted. Stretched health and care resources necessitate alternative and innovative ways to providing care for persons with dementia [18]. Assistive technology has been suggested as a means to support persons with dementia and their carers to stay independent in the community [19, 20]. Assistive technology (AT) can be defined as 'any item, piece of equipment, product or system that is used to increase, maintain or improve the functional capabilities and independence of people with cognitive, physical or communication difficulties' [21].

AT can be specialist equipment that is prescribed by a healthcare professional or available 'off-the shelf'. AT can be products such as clocks, medication dispensers, smartphone apps, robotic vacuum cleaners, smart gas meters, social assistive robots, communication books, navigation systems, falls and motion detectors and door exit alarms [22-24]. Use of AT by persons with dementia may by extension also benefit the carer [18, 25], which could potentially increase support from carers and alleviate some of the burden of caregiving [23, 2628]. AT potentially assists carers to address the increased level of responsibility whilst caring for a person with dementia [29]. Future generations will be more familiar with technology with people having lived their entire lives influenced by it. With advances in the Internet of Things and artificial intelligence, AT is likely to become more personalised to individual needs and user requirements [30]. Home is an important and special place when ageing. Living at home brings with it a sense of security and freedom [31, 32], and persons with dementia will strive to live at home for as long as possible. With technological advances, AT would be one way of assuring this continues to happen. Carers of persons with dementia are in the unique position of using their perceptions regarding AT to suggest or even decide on the access and use of AT [33, 34].

\section{Rationale for this review}

Currently, AT is being viewed as a panacea for reducing carer burden [32, 35]; multiple studies are investigating how AT can support persons with dementia [19, 36]. Carers could be using the AT together with the person with dementia and/or be looking after a person with dementia, who uses AT independently of the carer. However, little is known about the experiences of carers using AT and what impact AT has on carer outcomes [37]. This review aims to fill the gap in the literature that so far has predominantly looked at AT from the perspective of persons with dementia and its use within institutional settings $[18,38-40]$. We seek to answer research questions regarding experiences of informal carers using various AT and their effectiveness. This information would benefit carers and persons with dementia and healthcare professionals who prescribe and set up AT solutions at home as well as industry and AT developers. The results from the review will result in a summary matrix of common types (e.g. mobile phones, alarms) and uses (e.g. communication, safety, personal care) of AT in dementia care. This matrix will be further refined in a consensus meeting with stakeholders, including carers and health professionals who usually prescribe AT.

\section{Review aim and questions}

This review will aim to:

1) Identify the types and uses of AT in dementia

2) Describe the effectiveness of AT for burden, wellbeing and quality of life of informal carers of people with dementia living at home

3) Describe carers' experiences of AT use in dementia

4) Determine the aspects of AT that are valued and work well for carers by integrating (2) and (3) as above.

The following information will also be reported: What was the profile of informal carers and persons with 
dementia in the studies? What was the range of outcome measures used to report on burden, quality of life and wellbeing in the included studies? How is the caregiver/ care receiver relationship reported?

\section{Methods/design}

This protocol has been prepared following the Preferred Reporting Items for Systematic Review and Meta-Analysis Protocols (PRISMA-P) guidelines for systematic review protocols [41]. (The PRISMA-P checklist is included as Additional file 1). The review protocol has been registered with the International Prospective Register of Systematic Reviews PROSPERO (CRD42017082268).

This review will gather evidence on the effectiveness and informal carers' experience when using AT to support persons with dementia at home including perceived burden, quality of life and wellbeing with use of AT at home.

\section{Inclusion/exclusion criteria}

\section{Types of participants}

Studies that include informal carers who provide care for a person with dementia at home without being reimbursed will be included. Care could be supporting a person with dementia physically, emotionally, financially or socially and be provided by a relative, a friend or a neighbour of the person with dementia. There will be no restrictions regarding sex, living arrangements or ethnic background. Studies reporting on informal carers who provide support to a person with dementia receiving care in hospital and/or long-term institutions, informal carers who are younger than 18 years of age and formal/ paid carers will be excluded.

\section{Types of studies}

To answer the review questions, both quantitative and qualitative study designs will be included. Restriction will not be placed on the identification of studies by design or methods. The full-text article or report of the study needs to be available (i.e. abstracts will be excluded). We will not be including other reviews; however, we will check references within identified existing reviews on dementia, informal carers and AT to ensure that all relevant studies have been located. Letters to the editor, abstract and conference proceedings and book reviews will not be included. Study protocols and theses/ dissertations will also be excluded.

\section{Types of assistive technology}

For this review, studies that evaluate AT use in dementia involving informal carers will be included. AT will be defined as any advanced electronic equipment, which can be used to enhance support and care, act as a prompt for intervention by carers, monitor welfare and assist in communication and leisure activities for a person with dementia. This AT can be stand alone or be part of an integrated system and can be stationary or mobile. The focus of most research studies invariably is on the person with dementia, but any study that reports on effects or experiences of AT use on informal carers will be included. Studies that report solely on AT use for persons with dementia without including informal carers will be excluded, as will studies that focus only on electronic therapeutic interventions that are not AT (e.g. computer-based education or support for carers).

\section{Comparators}

For appropriate study designs, the control interventions could be 'care (or treatment) as usual' or non-technological or other nonpharmacological interventions such as monitoring and support at home by paid carers and interventions that use non-electronic assistive devices such as dosette boxes.

\section{Outcome measures}

Primary For evaluation of effectiveness, carer burden, quality of life, and wellbeing will be included. For evaluation of experience, all reported or observed experiences of usefulness, benefits and disadvantages of AT and impact on caregiver/care receiver relationship will be included.

Secondary For evaluation of effectiveness, carer selfesteem and feeling of competence and reported environmental changes to the home as a result of AT use will be considered. For evaluating experience, barriers and facilitators, user-friendliness and support required to use AT will be considered.

A summary of the criteria for inclusion and exclusion of studies for this systematic review is given in Additional file 4: Table S1.

\section{Search strategy}

The search strategy has been developed in collaboration with a Bodleian medical library librarian at the University of Oxford.

Searches will be carried out on:

\section{A. Databases}

The databases included were MEDLINE (Ovid) from 1946 to present, EMBASE from 1974 to present, PsyCINFO from 1806 to present, AMED 1985 to present, CINAHL from 1981 to present, Database of Abstracts of Reviews of Effects (DARE), OT seeker and The Cochrane Library of Systematic Reviews. The search will include studies within ALOIS (from database inception to present). ALOIS is maintained by the Trials Search Coordinator of the Cochrane Dementia Group and 
contains dementia and cognitive improvement studies identified from various databases and trial registries.

\section{B. Unpublished literature}

The International Standard Randomised Controlled Trials Number (ISRCTN) registry [42] and the National Institutes of Health Clinical Trials Database [43] will be searched for information on unpublished trials, and OpenGrey will be searched for conference papers, official publications and other types of grey literature. In addition, organisations within national and international settings, including AGE-WELL NCE (Aging Gracefully across Environments using Technology to Support Wellness, Engagement and Long Life) in Canada and Rehabilitation Engineering and Assistive Technology Society for North America (RESNA) will be contacted. Searches within these databases will be used to identify additional studies and authors to contact for full-text reports.

C. Manual searches of reference lists will also be conducted to identify relevant research studies.

Searches will not be limited by quantitative or qualitative filters, but due to funding constraints, only English language or those translated to English language will be included. We will however scan references of potential papers in languages other than English and list these in the review to provide a more complete picture of potential evidence, to inform future updates of the review. No date limits will be applied. The search strategy for the search on MEDLINE (Ovid) database is provided (Additional file 2).

\section{Screening}

Electronic search results will be downloaded into Covidence software [44]. Covidence is a web-based software platform that streamlines the production of systematic reviews. Duplicates will be removed using the software. Authors VS and MP will independently screen all titles and abstracts for eligibility against our inclusion/exclusion criteria. Any discrepancies will be resolved by mutual discussion. For studies that have insufficient information from the title and abstract, full text will be retrieved to determine inclusion. Studies marked for possible inclusion will undergo a full-text review. At full-text review, if both VS and MP agree that a study does not meet the full eligibility criteria, the study will be excluded. Any disagreement will be resolved by discussion and in consensus with the third author CJ. Reasons for exclusion of the full-text studies will be documented and listed separately.

\section{Data extraction}

A bespoke data extraction form (Additional file 3) developed by the authors will be used and initially piloted on a sample of studies to refine the form. Data will be extracted differently for the effectiveness and the experience evaluations from quantitative and qualitative studies respectively, and appropriate columns will be filled for mixed method studies.

\section{Evaluation of effectiveness}

Data extraction from quantitative studies will be based on the recommended items from the Cochrane handbook for systematic reviews of interventions [45]. Information on citation including authors and contact details, study design, duration, number of participants, setting, participant gender, age and ethnicity, country where the study took place, relationship status to the person with dementia, interventions, outcome measures or scaled used, time points of data collection, missing participants and key conclusions from the study authors will be extracted.

\section{Evaluation of experience}

In addition to collecting information from qualitative studies on citation, author contact details, study design, duration, setting, participant information, country and time points when information was collected, VS will extract data based on participants' quotes and study authors' commentaries. MP will check all extracted data for accuracy and completeness. Any disagreement will be resolved by discussion with $\mathrm{CJ}$.

\section{Risk of bias assessment}

The Mixed Methods Appraisal Tool [46-48] for assessing bias will be used for risk of bias assessment. This tool is designed for the appraisal stage of systematic mixed studies reviews, i.e. reviews that include qualitative, quantitative and mixed methods studies. The selected studies will be critically evaluated by VS and discussed with MP and CJ with any discrepancies resolved through discussion. Authors' conclusions on the quality of the included studies will be part of the summary of included studies.

\section{Data synthesis}

Where possible, quantitative results of studies will be pooled based on outcome measures and we will show forest plots and estimate pooled effects. Qualitative results will be synthesised using thematic synthesis [49]. Our preliminary searches and other reviews in AT have shown studies are heterogeneous; in this case, a descriptive summary and narrative synthesis [50] of the included studies will be provided. Where data is available, a subgroup analysis using chi-squared test for sub-group differences to assess for subgroup interactions based on device types, uses, living arrangements, adverse events and demographics of carers (country, age, gender, relationship, ethnicity etc.) will be done. VS will extract data and summarise the 
results. MP will review and highlight any discrepancies, and outstanding issues will be resolved through mutual discussion and involvement of $\mathrm{CJ}$ as necessary.

\section{Potential limitations}

We anticipate certain limitations to our review. Due to the prospect that there will be a widespread range of AT, reported outcomes and outcome measures used within the studies, there will be widespread heterogeneity in the included studies. Due to financial constraints, we are not including languages other than English within this review and this could potentially miss some suitable studies. We are also not including unpublished data within this systematic review due to the potential of low quality of data from non-peer-reviewed sources.

\section{Discussion}

The main aim of this review is to describe informal carers' perception and experience of AT use in dementia care. The results of the review are expected to inform a summary matrix of common types (e.g. mobile phones, alarms) and uses (e.g. communication, safety, personal care) of AT in dementia care. The review will also identify commonly used outcome measures that assess informal carer burden and quality of life in conjunction with AT use in dementia. The inclusion of quantitative and qualitative study designs will help understand the breadth and depth of informal carers' experience of AT use for a person with dementia. The review will highlight investigations on the impact of AT use on informal carers and commonly used outcome measures and experience questionnaires that have been used within this research area. The findings from this review will be published and disseminated (journals, conferences and social media), and it is anticipated that the findings will be useful for healthcare professionals, commissioners and AT developers to inform better provision of care in the community for carers of persons with dementia.

\section{Additional files}

Additional file 1: PRISMA-P checklist. (DOCX $35 \mathrm{~kb}$ )

Additional file 2: MEDLINE (OVID) search strategy for this review. (DOCX $13 \mathrm{~kb}$ )

Additional file 3: Data extraction form for this review. (DOCX $15 \mathrm{~kb}$ )

Additional file 4: Table of inclusion and exclusion criteria for this review. (DOCX $15 \mathrm{~kb}$ )

\footnotetext{
Abbreviations

ALOIS: ALOIS, named after Alois Alzheimer, is a register of dementia studies maintained by the Cochrane Dementia and Cognitive Improvement Group; AMED: Allied and Complementary Medicine Database; AT: Assistive technology; CINAHL: Cumulative Index of Nursing and Allied Health Literature; PRISMA-P: Preferred Reporting Items for Systematic Reviews and Meta-Analyses Protocols; PROSPERO: International Prospective Register of Systematic Review
}

\section{Acknowledgements}

The authors would like to acknowledge support from Ms. Nia Roberts (Medical library librarian) for the help with the search strategy. The authors also acknowledge the constructive comments from the reviewers and associate editor of this journal which have helped clarify, refine and strengthen this manuscript.

\section{Authors' contributions}

VS, CJ and MP conceived the design of the study. VS developed the search strategy together with a qualified librarian and conducted the search. VS and MP checked studies for eligibility, and CJ resolved any conflicts in study selection. VS drafted the protocol with critical revision and input from MP and CJ. All authors have read and given approval for the final version of the manuscript. VS is the guarantor of the review.

\section{Authors' information \\ VS is a postgraduate student registered for his DPhil at the University of Oxford exploring informal carers' experience of assistive technology use in dementia. MP is a senior researcher within the Health Services Research Unit (HSRU), Nuffield Department of Population Health, University of Oxford. CJ is Professor of Health Services Research and Director of the HSRU, Nuffield Department of Population Health, University of Oxford. MP and CJ are joint supervisors of VS for the DPhil.}

\section{Funding}

This systematic review is part of a DPhil in Population Health at the University of Oxford.

\section{Availability of data and materials}

Not applicable.

Ethics approval and consent to participate

Not applicable.

\section{Consent for publication}

Not applicable.

\section{Competing interests}

The authors declare that they have no competing interests.

Received: 27 March 2018 Accepted: 28 June 2019

Published online: 03 July 2019

\section{References}

1. World Health Organization. Dementia: a public health priority. Geneva: World Health Organization; 2012. http://www.who.int/mental_health/ publications/dementia_report_2012/en/. Accessed 27 Nov 2017

2. Prince $M$, Wimo A, Guerchet $M$, Ali G-C, Wu Y-T, Prina $M$, et al. The global impact of dementia: an analysis of prevalence, incidence, cost and trends. London; 2015. https://doi.org/10.1111/j.0963-7214.2004.00293.x.

3. Wilson MR, Van Houtven CH, Stearns SC, Clipp EC. Depression and missed work among informal caregivers of older individuals with dementia. J Fam Econ Issues. 2007;28:684-98. https://doi.org/10.1007/s10834-007-9081-8.

4. Lewis F, Karlsberg Schaffer S, Sussex J, O'Neill P, Cockcroft L. The trajectory of dementia in the UK - making a difference. London; 2014. https://www. ohe.org/publications/trajectory-dementia-uk-making-difference\#. Accessed 27 Nov 2017

5. Luengo-Fernandez R, Leal J, Gray A. The economic burden of dementia and associated research funding in the United Kingdom. Cambridge; 2010. https://www.scie-socialcareonline.org.uk/dementia-2010-the-economicburden-of-dementia-and-associated-research-funding-in-the-unitedkingdom/r/a11G00000017sGjlAl. Accessed 27 Nov 2017.

6. Lancet T. Addressing global dementia. Lancet (London, England). 2014;383: 2185. https://doi.org/10.1016/S0140-6736(14)61066-7.

7. Adult Social Care Statistics Team. Personal social services survey of adult carers in England, 2016-17 - NHS Digital. London; 2017. https://digital.nhs. uk/catalogue/PUB30045. Accessed 27 Nov 2017.

8. Skills for Care, Dementia UK. Dementia: workers and carers together: a guide for social care workers on supporting family and friends carers of people with dementia. 2012;34. http://www.skillsforcare.org.uk/Document-library/Skills/ Dementia/Dandctog-final-versionndd.pdf. Accessed 27 Nov 2017. 
9. Sansoni J, Vellone E, Piras G. Anxiety and depression in communitydwelling, Italian Alzheimer's disease caregivers. Int J Nurs Pract. 2004;10:93100. https://doi.org/10.1111/j.1440-172X.2003.00461.X.

10. Aguglia E, Onor ML, Trevisiol M, Negro C, Saina M, Maso E. Stress in the caregivers of Alzheimer's patients: an experimental investigation in Italy. Am J Alzheimer's Dis Other Dementiasr. 2004;19:248-52. https://doi.org/10.11 77/153331750401900403

11. Trivedi D, Goodman C, Dickinson A, Gage H, McLaughlin J, Manthorpe J, et al. A protocol for a systematic review of research on managing behavioural and psychological symptoms in dementia for community-dwelling older people: evidence mapping and syntheses. Syst Rev. 2013;2:70. https://doi. org/10.1186/2046-4053-2-70.

12. Manthorpe J, Bowling A. Quality of life measures for carers for people with dementia: measurement issues, gaps in research and promising paths. Res Policy Plan. 2016;31:163-78. http://ssrg.org.uk/members/files/2015/07/ Manthorpe-Bowling.pdf. Accessed 2 Nov 2017.

13. Abdollahpour I, Nedjat S, Noroozian M, Salimi Y, Majdzadeh R. Positive aspects of caregiving questionnaire. J Geriatr Psychiatry Neurol. 2017;30:7783. https://doi.org/10.1177/0891988716686831

14. Cross-Government publication. Recognised, valued and supported: next steps for the carers strategy. London; 2010. https://www.gov.uk/ government/uploads/system/uploads/attachment_data/file/213804/ dh_122393.pdf. Accessed 29 Nov 2017.

15. NHS England. NHS England »Commitment to carers. NHS England. 2014;:1. https://www.england.nhs.uk/commitment-to-carers/. Accessed 29 Nov 2017.

16. NHS England. NHS England » NHS England Chief Executive committed to improving dementia diagnosis. NHS England. 2014;:1. https://www.england. nhs.uk/2014/09/dementia-guide/. Accessed 29 Nov 2017.

17. Care Act. Care Act 2014. Statute Law Database; 2014. http://www.legislation. gov.uk/ukpga/2014/23/contents. Accessed 29 Nov 2017.

18. Daly Lynn J, Rondón-Sulbarán J, Quinn E, Ryan A, McCormack B, Martin S. A systematic review of electronic assistive technology within supporting living environments for people with dementia. Dementia.. 2017;147130121773364. doi:https://doi.org/10.1177/1471301217733649.

19. Gibson G, Dickinson C, Brittain K, Robinson L. The everyday use of assistive technology by people with dementia and their family carers: a qualitative study. BMC Geriatr. 2015;15:1-10. https://doi.org/10.1186/s12877-015-0091-3.

20. Boyd H, Evans N, Cheston R, Noonan K, Harris N. Home testing of a digital prompter for people with dementia. Stud Health Technol Inform. 2017;242:2730. http://www.ncbi.nlm.nih.gov/pubmed/28873772. Accessed 27 Oct 2017.

21. The Audit commission. Assistive technology. London; 2004. http://www.wales. nhs.uk/documents/National20Report_FINAL.pdf. Accessed 27 Nov 2017

22. Gibson G, Newton L, Pritchard G, Finch T, Brittain K, Robinson L. The provision of assistive technology products and services for people with dementia in the United Kingdom. Dementia. 2016;15:681-701. https://doi. org/10.1177/1471301214532643.

23. AKTIVE Consortium. The role of telecare in meeting the care needs of older people: themes, debates and perspectives in the literature on ageing and technology. Leeds; 2013. www.aktive.org.uk/publications.html. Accessed 27 Nov 2017

24. Paiva S, Abreu C. Low cost GPS tracking for the elderly and Alzheimer patients. Procedia Technol. 2012;5:793-802. https://doi.org/10.1016/J. PROTCY.2012.09.088.

25. Sörensen $S$, Pinquart $M$, Duberstein P. How effective are interventions with caregivers? An updated meta-analysis. Gerontologist. 2002;42:356-72 http:// www.ncbi.nlm.nih.gov/pubmed/12040138. Accessed 29 Nov 2017.

26. Bledsoe LK, Moore SE, Collins WL, Bledsoe LK, Moore SE, Collins WL, et al. Long distance caregiving: an evaluative review of the literature context for provision of care from a distance. Ageing Int. 2010;35:293-310. https://doi.org/10.1007/ s12126-010-9062-3.

27. Czaja SJ, Rubert MP. Telecommunications technology as an aid to family caregivers of persons with dementia. Psychosom Med. 2002;64:469-76. https://doi.org/10.1097/00006842-200205000-00011.

28. McKechnie V, Barker C, Stott J. Effectiveness of computer-mediated interventions for informal carers of people with dementia-a systematic review. Int Psychogeriatrics. 2014;26:1619-37. https://doi.org/10.1017/S1 041610214001045 .

29. Huschilt J, Clune L. The use of socially assistive robots for dementia care. J Gerontol Nurs. 2012;38:15-9. https://doi.org/10.3928/00989134-20120911-02.

30. National Institute for Health Research. Internet of Things being used in innovative dementia research: National Institute for Health Research; 2017. https://www.nihr.ac.uk/news/internet-of-things-being-used-in-innovativedementia-research/7247. Accessed 1 Dec 2017

31. Greenhalgh T, Wherton J, Sugarhood P, Hinder S, Procter R, Stones R. What matters to older people with assisted living needs? A phenomenological analysis of the use and non-use of telehealth and telecare. Soc Sci Med. 2013;93:86-94. https://doi.org/10.1016/J.SOCSCIMED.2013.05.036.

32. Mahoney DF, Purtilo RB, Webbe FM, Alwan M, Bharucha AJ, Adlam TD, et al. In-home monitoring of persons with dementia: ethical guidelines for technology research and development. Alzheimer's Dement. 2007;3:217-26. https://doi.org/10.1016/J.JALZ.2007.04.388.

33. Arntzen C, Holthe T, Jentoft R. Tracing the successful incorporation of assistive technology into everyday life for younger people with dementia and family carers. Dementia. 2016;15:646-62. https://doi.org/10.1177/14713 01214532263.

34. Fleming R, Sum S. Empirical studies on the effectiveness of assistive technology in the care of people with dementia: a systematic review. J Assist Technol. 2014;8:14-34. https://doi.org/10.1108/JAT-09-2012-0021.

35. Kang HG, Mahoney DF, Hoenig H, Hirth VA, Bonato P, Hajjar I, et al. In situ monitoring of health in older adults: technologies and issues. J Am Geriatr Soc. 2010;58:1579-86. https://doi.org/10.1111/j.1532-5415.2010.02959.x.

36. Kamel Boulos MN, Lou RC, Anastasiou A, Nugent CD, Alexandersson J, Zimmermann $\mathrm{G}$, et al. Connectivity for healthcare and well-being management: examples from six European projects. Int J Environ Res Public Health. 2009;6:1947-71. https://doi.org/10.3390/ijerph6071947.

37. Van der Roest HG, Wenborn J, Pastink C, Dröes R-M, Orrell M. Assistive technology for memory support in dementia. Cochrane Database Syst Rev. 2017. https://doi.org/10.1002/14651858.CD009627.pub2.

38. Gagnon-Roy M, Bourget A, Stocco S, Courchesne A-CL, Kuhne N, Provencher $\mathrm{V}$. Assistive technology addressing safety issues in dementia: a scoping review. Am J Occup Ther. 2017;71:7105190020p1. https://doi.org/1 0.5014/ajot.2017.025817.

39. Brims L, Oliver K. Effectiveness of assistive technology in improving the safety of people with dementia: a systematic review and meta-analysis. Aging Ment Health. 2018:1-10. https://doi.org/10.1080/13607863.2018.1455805.

40. Khosravi P, Ghapanchi AH. Investigating the effectiveness of technologies applied to assist seniors: a systematic literature review. Int J Med Inform. 2016;85:17-26. https://doi.org/10.1016/J.IJMEDINF.2015.05.014.

41. Shamseer L, Moher D, Clarke M, Ghersi D, Liberati A, Petticrew M, et al. Preferred reporting items for systematic review and meta-analysis protocols (PRISMA-P) 2015: elaboration and explanation. BMJ. 2015;350:g7647. https:// doi.org/10.1136/BMJ.G7647.

42. International Standard Randomised Controlled Trials Number. ISRCTN Registry. https://www.isrctn.com/. Accessed 19 Jan 2019.

43. National Institute of Health. ClinicalTrials.gov. https://clinicaltrials.gov/. Accessed 19 Jan 2019

44. Veritas Health Innovation. Covidence systematic review software. 2017. www.covidence.org.

45. Higgins J, Green S. Cochrane handbook for systematic reviews of interventions version 5.1.0. 2011. http://handbook.cochrane.org.

46. Crowe M, Sheppard L. A review of critical appraisal tools show they lack rigor: alternative tool structure is proposed. J Clin Epidemiol. 2011;64:79-89. https://doi.org/10.1016/j.jclinepi.2010.02.008.

47. Pluye P, Gagnon MP, Griffiths F, Johnson-Lafleur J. A scoring system for appraising mixed methods research, and concomitantly appraising qualitative, quantitative and mixed methods primary studies in Mixed Studies Reviews. Int J Nurs Stud. 2009;46:529-46. https://doi.org/10.1016/j. ijnurstu.2009.01.009.

48. Pluye P, Hong QN. Combining the power of stories and the power of numbers: mixed methods research and mixed studies reviews. Annu Rev Public Health. 2014;35:29-45. https://doi.org/10.1146/annurev-publhealth032013-182440.

49. Timulak $L$. The SAGE handbook of qualitative data analysis. London: SAGE publications Ltd; 2015. https://uk.sagepub.com/en-gb/eur/the-sagehandbook-of-qualitative-data-analysis/book237405. Accessed 28 Aug 2018

50. Thomas J, Harden A. Methods for the thematic synthesis of qualitative research in systematic reviews. BMC Med Res Methodol. 2008;8:45. https:// doi.org/10.1186/1471-2288-8-45.

\section{Publisher's Note}

Springer Nature remains neutral with regard to jurisdictional claims in published maps and institutional affiliations. 